\title{
ON THE LIMIT CYCLES OF A CLASS OF PIECEWISE LINEAR DIFFERENTIAL SYSTEMS IN $\mathbb{R}^{4}$ WITH TWO ZONES
}

\author{
CLAUDIO A. BUZZI ${ }^{1}$, JAUME LLIBRE ${ }^{2}$ AND JOÃO C. MEDRADO ${ }^{3}$
}

\begin{abstract}
We study the bifurcation of limit cycles from the periodic orbits of a four-dimensional center in a class of piecewise linear differential systems with two zones. Our main result shows that three is an upper bound for the number of limit cycles that bifurcate from a center, up to first order expansion of the displacement function. Moreover, this upper bound is reached. The main technique used is the averaging method.
\end{abstract}

\section{INTRODUCTION AND STATEMENT OF THE MAIN RESUlT}

In the qualitative theory of differential equations the study of their limit cycles became one of the main topics. For a given differential system a limit cycle is a periodic orbit isolated in the set of all periodic orbits of the system.

Many questions arise on the limit cycles of the planar differential equations. Two main lines of research for such equations are, first the 16th Hilbert problem, see for instance [6, 7], and second the study of how many limit cycles emerge from the periodic orbits of a centre when we perturb it inside a given class of differential equations, see, for example, the book [3] and the references there in. More precisely, the problem of considering the planar linear differential centre

$$
\dot{x}=-y, \quad \dot{y}=x
$$

and perturbing it

$$
\dot{x}=-y+\varepsilon P(x, y), \quad \dot{y}=x+\varepsilon Q(x, y),
$$

inside a given class of polynomial differential equations and studying the limit cycles bifurcating from the periodic orbits of the linear centre has attracted the interest and the research of many mathematicians. Of course, $\varepsilon$ is a small parameter. Here our main concerning is to bring this problem to higher dimension when the perturbation is piecewise linear.

In [9] Lum and Chua conjectured that a continuous piecewise linear vector field in the plane with two zones has at most one limit cycle. Moreover if the limit cycle exists, then it is either attracting or repelling. This conjecture was proved in [4].

The purpose of this paper is to study the problem of Lum and Chua for a class of piecewise linear differential systems in dimension 4 with two zones, more precisely to study the maximum number of limit cycles of the 4-dimensional continuous piecewise linear vector fields with two zones of the form

$$
x^{\prime}=A_{0} x+\varepsilon F(x)
$$

1991 Mathematics Subject Classification. 58F14, 58F21, 58F30.

Key words and phrases. limit cycles, averaging theory, piecewise linear systems with two zones. 
for $|\varepsilon| \neq 0$ a sufficiently small real parameter, where

$$
A_{0}=\left(\begin{array}{cccc}
0 & -1 & 0 & 0 \\
1 & 0 & 0 & 0 \\
0 & 0 & 0 & -1 \\
0 & 0 & 1 & 0
\end{array}\right),
$$

and $F: \mathbb{R}^{4} \rightarrow \mathbb{R}^{4}$ is given by

$$
F(x)=A x+\varphi\left(k^{T} x\right) b,
$$

with $A \in \mathcal{M}_{4}(\mathbb{R}), k, b \in \mathbb{R}^{4} \backslash\{0\}$ and $\varphi: \mathbb{R} \rightarrow \mathbb{R}$ the piecewise linear function

$$
\varphi(x)= \begin{cases}0 & \text { for } x \in(-\infty, 1) \\ m x & \text { for } x \in[1, \infty)\end{cases}
$$

For $\varepsilon=0$ system (1) becomes

$$
x_{1}^{\prime}=-x_{2}, x_{2}^{\prime}=x_{1}, x_{3}^{\prime}=-x_{4}, x_{4}^{\prime}=x_{3} .
$$

Our main result is the following.

Theorem 1. Three is the upper bound for the number of limit cycles of system (1) which bifurcate from the periodic orbits of system (3), up to first order expansion of the displacement function of (1) with respect to the small parameter $\varepsilon$. Moreover, there are systems (1) having three limit cycles.

Theorem 1 is proved in section 3 . In section 2 we present the results from the averaging theory necessary for proving Theorem 1 .

In [1] it has been study a similar problem for 4-dimensional continuous piecewise linear vector fields but with three zones having symmetry respect to the origin. In fact those piecewise linear vector fields are relevant in control theory.

\section{Averaging Theory}

The aim of this section is to present the first order averaging method as it was obtained in Buică \& Llibre [2]. Differentiability of the vector field is not needed. The specific conditions for the existence of a simple isolated zero of the averaged function are given in terms of the Brouwer degree. In fact, the Brouwer degree theory is the key point in the proof of this theorem. We remind here that continuity of some finite dimensional function is a sufficient condition for the existence of its Brouwer degree (see [8] for precise definitions). For a proof of the next result see for instance $[5,10,11]$.

Theorem 2. We consider the following differential system

$$
x^{\prime}(t)=\varepsilon H(t, x)+\varepsilon^{2} R(t, x, \varepsilon),
$$

where $H: \mathbb{R} \times D \rightarrow \mathbb{R}^{n}, R: \mathbb{R} \times D \times\left(-\varepsilon_{f}, \varepsilon_{f}\right) \rightarrow \mathbb{R}^{n}$ are continuous functions, $T$ periodic in the first variable, and $D$ is an open subset of $\mathbb{R}^{n}$. We define $h: D \times \mathbb{R}^{n}$ as

$$
h(z)=\int_{0}^{T} H(s, z) d s
$$

and assume that:

(i) $H$ and $R$ are locally Lipschitz with respect to $x$; 
(ii) for $a \in D$ with $h(a)=0$, there exists a neighborhood $V$ of a such that $h(z) \neq 0$ for all $z \in \bar{V} \backslash\{a\}$ and $d_{B}(h, V, a) \neq 0$.

Then, for $|\varepsilon|>0$ sufficiently small, there exists an isolated T-periodic solution $\varphi(\cdot, \varepsilon)$ of system (4) such that $\varphi(0, \varepsilon) \rightarrow a$ as $\varepsilon \rightarrow 0$.

Here we will need some facts from the proof of Theorem 2. Hypothesis (i) assures the existence and uniqueness of the solution of each initial value problem on the interval $[0, T]$. Hence, for each $z \in D$, it is possible to denote by $x(\cdot, z, \varepsilon)$ the solution of (4) with the initial value $x(0, z, \varepsilon)=z$. We consider also the function $\zeta: D \times\left(-\varepsilon_{f}, \varepsilon_{f}\right) \rightarrow \mathbb{R}^{n}$ defined by

$$
\zeta(z, \varepsilon)=\int_{0}^{T}\left[\varepsilon H(t, x(t, z, \varepsilon))+\varepsilon^{2} R(t, x(t, z, \varepsilon), \varepsilon)\right] d t .
$$

¿From the proof of Theorem 2 we extract the following facts.

Remark 3. For every $z \in D$ the following relation holds

$$
x(T, z, \varepsilon)-x(0, z, \varepsilon)=\zeta(z, \varepsilon) .
$$

The function $\zeta$ can be written in the form

$$
\zeta(z, \varepsilon)=\varepsilon h(z)+\varepsilon^{2} \mathcal{O}(1)
$$

where $h$ is given by $(5)$ and the symbol $\mathcal{O}(1)$ denotes a bounded function on every compact subset of $D \times\left(-\varepsilon_{f}, \varepsilon_{f}\right)$. Moreover, for $|\varepsilon|$ sufficiently small, $z=\varphi(0, \varepsilon)$ is an isolated zero of $\zeta(\cdot, \varepsilon)$.

For concrete systems there is the possibility that the function $\zeta$ is not globally differentiable, but the function $h$ is. In fact, only differentiability in some neighborhood of a fixed isolated zero of $h$ could be enough. When this is the case, one can use the following remark in order to verify the hypothesis $(i i)$ of Theorem 2.

Remark 4. Let $h: D \rightarrow \mathbb{R}^{n}$ be a $\mathcal{C}^{1}$ function, with $h(a)=0$, where $D$ is an open subset of $\mathbb{R}^{n}$ and $a \in D$. Whenever $a$ is a simple zero of $h$ (i.e. $\left.J h(a) \neq 0\right)$, there exists a neighborhood $V$ of a such that $h(z) \neq 0$ for all $z \in \bar{V} \backslash\{a\}$. Then $d_{B}(h, V, a) \in\{-1,1\}$.

\section{Proof of Theorem 1}

First we show that instead of working with the function $\varphi\left(k^{T} x\right)$ in system (1) we can work without loss of generality with an easier function, namely $\varphi\left(x_{1}\right)$, where $x=\left(x_{1}, \ldots, x_{4}\right)$.

Lemma 5. By a linear change of variables, and eventually a permutation of the variables, system (1) can be transformed in the system

$$
x^{\prime}=A_{0} x+\varepsilon \bar{A} x+\varepsilon \varphi\left(x_{1}\right) \bar{b}
$$

where $\bar{A}=\left(a_{i j}\right)$ and $\bar{b}=\left(b_{i}\right)$.

Proof. A linear change of coordinates $x=J y$, with $J$ invertible, transforms system (1) in

$$
\dot{y}=J^{-1} A_{0} J y+\varepsilon J^{-1} A J y+\varepsilon \varphi\left(k^{T} J y\right) J^{-1} b .
$$

It is easy find $J$ invertible that satisfies $J^{-1} A_{0} J=A_{0}$ and $k^{T} J=e_{1}^{T}$. 
In order to apply the averaging method, we put the system in a more suitable form. It is easy to see that the change of variables $\left(x_{1}, x_{2}, x_{3}, x_{4}\right)$ to $(\theta, r, \rho, s)$ by

$$
\begin{aligned}
x_{1}=r \cos \theta, & x_{2}=r \sin \theta, \\
x_{3}=\rho \cos (\theta+s), & x_{4}=\rho \sin (\theta+s),
\end{aligned}
$$

transforms system (7) into a system of the form

$$
\begin{aligned}
& \frac{d r}{d \theta}=\varepsilon H_{1}(\theta, r, \rho, s)+\varepsilon^{2} \mathcal{O}(1) \\
& \frac{d \rho}{d \theta}=\varepsilon H_{2}(\theta, r, \rho, s)+\varepsilon^{2} \mathcal{O}(1) \\
& \frac{d s}{d \theta}=\varepsilon H_{3}(\theta, r, \rho, s)+\varepsilon^{2} \mathcal{O}(1)
\end{aligned}
$$

for $H_{1}, H_{2}$ and $H_{3}$ given by

$$
\begin{aligned}
H_{1}= & \cos \theta\left[b_{1} \varphi(r \cos \theta)+a_{11} r \cos \theta+a_{12} r \sin \theta+a_{13} \rho \cos \tilde{\theta}+a_{14} \rho \sin \tilde{\theta}\right]+ \\
& \sin \theta\left[b_{2} \varphi(r \cos \theta)+a_{21} r \cos \theta+a_{22} r \sin \theta+a_{23} \rho \cos \tilde{\theta}+a_{24} \rho \sin \tilde{\theta}\right], \\
H_{2}= & \cos \tilde{\theta}\left[b_{3} \varphi(r \cos \theta)+a_{31} r \cos \theta+a_{32} r \sin \theta+a_{33} \rho \cos \tilde{\theta}+a_{34} \rho \sin \tilde{\theta}\right]+ \\
& \sin \tilde{\theta}\left[b_{4} \varphi(r \cos \theta)+a_{41} r \cos \theta+a_{42} r \sin \theta+a_{43} \rho \cos \tilde{\theta}+a_{44} \rho \sin \tilde{\theta}\right], \\
H_{3}= & (1 / r)\left\{\sin \theta\left[b_{1} \varphi(r \cos \theta)+a_{11} r \cos \theta+a_{12} r \sin \theta+a_{13} \rho \cos \tilde{\theta}+a_{14} \rho \sin \tilde{\theta}\right]+\right. \\
& \left.\cos \theta\left[b_{2} \varphi(r \cos \theta)+a_{21} r \cos \theta+a_{22} r \sin \theta+a_{23} \rho \cos \tilde{\theta}+a_{24} \rho \sin \tilde{\theta}\right]\right\}- \\
& (1 / \rho)\left\{\sin \tilde{\theta}\left[b_{3} \varphi(r \cos \theta)+a_{31} r \cos \theta+a_{32} r \sin \theta+a_{33} \rho \cos \tilde{\theta}+a_{34} \rho \sin \tilde{\theta}\right]+\right. \\
& \left.\cos \tilde{\theta}\left[b_{4} \varphi(r \cos \theta)+a_{41} r \cos \theta+a_{42} r \sin \theta+a_{43} \rho \cos \tilde{\theta}+a_{44} \rho \sin \tilde{\theta}\right]\right\},
\end{aligned}
$$

where $\tilde{\theta}=\theta+s$.

Next step is apply Theorem 2 obtaining $h: D_{n} \rightarrow \mathbb{R}^{3}, h=\left(h_{1}, h_{2}, h_{3}\right)$ where

$$
h_{i}(r, \rho, s)=\int_{0}^{2 \pi} H_{i}(\theta, r, \rho, s) d \theta .
$$

We have

$$
\begin{aligned}
h_{1}(r, \rho, s)= & b_{1} I_{1}(r)+\pi\left(c_{1} r+c_{2} \rho \cos s+c_{3} \rho \sin s\right) \\
h_{2}(r, \rho, s)= & I_{1}(r)\left(b_{3} \cos s+b_{4} \sin s\right)+\pi\left(c_{4} \rho+c_{5} r \cos s+c_{6} r \sin s\right) \\
h_{3}(r, \rho, s)= & I_{1}(r)\left(-b_{2} \rho+b_{4} r \cos s-b_{3} r \sin s\right)+ \\
& \pi\left(c_{7} r \rho+c_{6} r^{2} \cos s+c_{5} r^{2} \sin s+c_{3} \rho^{2} \cos s-c_{2} \rho^{2} \sin s\right)
\end{aligned}
$$

where $c_{i}$ are constants that depend linearly on $a_{i j}$ and

$$
I_{1}(r)= \begin{cases}-m \frac{\sqrt{r^{2}-1}}{r}+m \arctan \left(\sqrt{r^{2}-1}\right) & \text { if } r \geq 1, \\ 0 & \text { if } 0<r<1 .\end{cases}
$$

Next lemma will be useful in the sequel of the proof. It was proved in [1] but since it is proof is easy we provide it for completeness.

Lemma 6. The equation $I_{1}(r)=c r$ is such that

(1) If $c / m<0$ or $c / m \geq \pi / 2$ then the equation does not have solution.

(2) If $c=0$ then the interval $(0,1]$ is a continuous of solutions.

(3) If $0<c / m<\pi / 2$ then there is an unique solution $r^{*}>1$. 
Proof. If $c=0$ then it is easy to see that all $r \in(0,1]$ is solution. For $c \neq 0$ we consider the change of variables $u=\sqrt{r^{2}-1}$ and we obtain the equivalent equation

$$
\arctan (u)=\frac{u}{1+u^{2}}+\frac{c}{m} \text {. }
$$

A simple graphical analysis shows that for $0<c / m<\pi / 2$ we have only one solution $u^{*}>0$ that corresponds to $r^{*}>1$.

Solving the two first equations of (9) we get

$$
I_{1}(r)=\frac{k_{2}(s)}{d(s)} r, \quad \rho=\frac{k_{1}(s)}{d(s)} r
$$

where $k_{1}(s)=\left(b_{1} c_{6}-b_{4} c_{1}\right) \sin s+\left(b_{1} c_{5}-b_{3} c_{1}\right) \cos s, k_{2}(s)=\pi\left(c_{1} c_{4}-c_{3} c_{6} \sin ^{2} s-\right.$ $\left.\left(c_{3} c_{5}+c_{2} c_{6}\right) \sin s \cos s-c_{2} c_{5} \cos ^{2} s\right)$ and $d(s)=-b_{1} c_{4}+b_{4} c_{3} \sin ^{2} s+\left(b_{4} c_{2}+b_{3} c_{3}\right) \sin s \cos s+$ $b_{3} c_{2} \cos ^{2} s$.

Substituting in to the third equation we obtain

$$
\frac{f(s)}{d(s)^{2}} r^{2}=0,
$$

where $f(s)=\left(c_{3} \pi \cos s-c_{2} \pi \sin s\right)\left(k_{1}(s)\right)^{2}+\left(b_{4} \cos s-b_{3} \sin s\right) d(s) k_{2}(s)-b_{2} k_{1}(s) k_{2}(s)+$ $\left(c_{6} \pi \cos s-c_{5} \pi \sin s\right)(d(s))^{2}+c_{7} \pi d(s) k_{1}(s)$.

Observe that if $s^{*}$ is a solution of $f(s)=0$, then it can be related to a periodic solution of system (1) only if it satisfies

$$
d\left(s^{*}\right) \neq 0, \frac{k_{1}\left(s^{*}\right)}{d\left(s^{*}\right)}>0 \text { and } 0<\frac{k_{2}\left(s^{*}\right)}{d\left(s^{*}\right)}<\frac{\pi}{2} .
$$

In this case we apply Lemma 6 and obtain $r^{*}>1$. And the second equation of (9) gives $\rho^{*}$.

Now we study the maximum number of solutions of the equation $f(s)=0$. Substituting $\cos s=x$ and $\sin s=\sqrt{1-x^{2}}$ in $f(s)=0$ we get

$$
\left(\delta_{1} x+\delta_{2} x^{3}\right)+\left(\delta_{3}+\delta_{4} x^{2}\right) \sqrt{1-x^{2}}=0,
$$

and substituting $\cos s=x$ and $\sin s=-\sqrt{1-x^{2}}$ in $f(s)=0$ we get

$$
\left(\delta_{1} x+\delta_{2} x^{3}\right)-\left(\delta_{3}+\delta_{4} x^{2}\right) \sqrt{1-x^{2}}=0 .
$$

Equations (10) and (11) are equivalent to

$$
\left(\delta_{1} x+\delta_{2} x^{3}\right)^{2}-\left(\delta_{3}+\delta_{4} x^{2}\right)^{2}\left(1-x^{2}\right)=0,
$$

which has degree six. Observe that the solutions appear in pairs $\left\{x_{0},-x_{0}\right\}$. So if $s^{*}$ is a solution then $s^{*}+\pi$ also is.

The functions $f(s), d(s), k_{1}(s)$ and $k_{2}(s)$ have the properties $f(s+\pi)=-f(s)$, $d(s+\pi)=d(s), k_{1}(s+\pi)=-k_{1}(s)$ and $k_{2}(s+\pi)=k_{2}(s)$. So we have

$$
\frac{k_{1}\left(s^{*}\right)}{d\left(s^{*}\right)}>0 \Rightarrow \frac{k_{1}\left(s^{*}+\pi\right)}{d\left(s^{*}+\pi\right)}<0
$$

We conclude that the maximum number of limit cycles for system (1), up to first order expansion of the displacement function, is three. 
In order to show that there exists an example with exactly three limit cycles we consider the values

$$
\begin{array}{lllll}
a_{11}=-13, & a_{12}=0, & a_{13}=0, & a_{14}=1886, & b_{1}=1, \\
a_{21}=0.29, & a_{22}=0, & a_{23}=0, & a_{24}=0, & b_{2}=0, \\
a_{31}=-0.006, & a_{32}=0.02, & a_{33}=1, & a_{34}=0, & b_{3}=0, \\
a_{41}=0, & a_{42}=0, & a_{43}=0, & a_{44}=0, & b_{4}=0 .
\end{array}
$$

Computing the six solutions of $f(s)=0$ we get $\{0, \pi / 4, \pi / 2, \pi, 5 \pi / 4,3 \pi / 2\}$. And the values of $d(s), k_{1}(s)$ and $k_{2}(s)$ are given in the following table.

\begin{tabular}{|c|c|c|c|}
\hline$s^{*}$ & $d\left(s^{*}\right)$ & $k_{1}\left(s^{*}\right)$ & $k_{2}\left(s^{*}\right)$ \\
\hline 0 & -1 & -0.0068 & $-13 \pi$ \\
\hline$\pi / 4$ & -1 & -0.021 & $-4.87 \pi$ \\
\hline$\pi / 2$ & -1 & -0.02 & $-0.43 \pi$ \\
\hline$\pi$ & -1 & 0.0068 & $-13 \pi$ \\
\hline $5 \pi / 4$ & -1 & 0.021 & $-4.87 \pi$ \\
\hline $3 \pi / 2$ & -1 & 0.02 & $-0.43 \pi$ \\
\hline
\end{tabular}

The three solutions $s^{*}$ that satisfy $k_{1}\left(s^{*}\right) / d\left(s^{*}\right)>0$ and $k_{2}\left(s^{*}\right) / d\left(s^{*}\right)>0$ are $\{0, \pi / 4, \pi / 2\}$.

The values of solutions $s^{*}, r^{*}$ and $\rho^{*}$ and the value of the Jacobian at the solution $J h\left(r^{*}, \rho^{*}, s^{*}\right)$ are given in the following table.

\begin{tabular}{|c|c|c|c|}
\hline$s^{*}$ & $r^{*}$ & $\rho^{*}$ & $J h\left(r^{*}, \rho^{*}, s^{*}\right)$ \\
\hline 0 & 9.53 & 0.065 & -996.98 \\
\hline$\pi / 4$ & 1.78 & 0.037 & 354 \\
\hline$\pi / 2$ & 1.09 & 0.02 & 69 \\
\hline
\end{tabular}

Acknowledgments. We would like to thank the dynamical system research group of IMECC/UNICAMP for the hospitality offered to us during the preparation of part of this paper.

The first author is partially supported by a FAPESP-BRAZIL grant 07/06896-5. The first and the third authors are partially supported by CNPq and CAPES. The second author is partially supported by a MEC/FEDER grant number MTM200803437, by a CICYT grant number 2009SGR 410 and by ICREA Academia. All authors are also supported by the joint project CAPES-MECD grant PHB-20090025-PC and AUXPE-DGU 15/2010.

\section{REFERENCES}

[1] A. Buiç̆ J. Llibre, Bifurcation of limit cycles from a four-dimensional center in control systems. Internat. J. Bifur. Chaos Appl. Sci. Engrg. 15 (2005), 2653-2662.

[2] A. Buic $\breve{A}$ and J. Llibre, Averaging methods for finding periodic orbits via Brower degree, Bull. Sci. Math, 128 (2004), 7-22.

[3] C. Christopher and C. Li, Limit Cycles of Differential Equations, Advanced Courses in Mathematics CRM Barcelona, Birkhauser Verlag, Basel, 2007.

[4] E. Freire; E. Ponce; F. Rodrigo; F. Torres, Bifurcation sets of continuous piecewise linear systems with two zones. Internat. J. Bifur. Chaos Appl. Sci. Engrg. 8 (1998), 2073-2097.

[5] J. Guckenheimer and P. Holmes, Nonlinear oscillations, dynamical systems, and bifurcation of vector fields, Springer, 1983.

[6] Y.S. Ilyashenko, Centennial history of Hilbert's 16th problem, Bull. Amer. Math. Soc. 39 (2002), 301-354.

[7] J. Li, Hilbert's 16th problem and bifurcations of planar polynomial vector fields, Internat. J. Bifur. Chaos Appl. Sci. Eng. 13 (2003), 47-106. 
[8] N.G. Lloyd, Degree Theory, Cambridge University Press, 1978.

[9] R. Lum and L.O. Chua, Global properties of continuous piecewise-linear vector fields. Part I: Simplest case in $R^{2}$, Memorandum UCB/ERL M90/22, University of California at Berkeley, 1990.

[10] J.A. Sanders and F. Verhulst, Averaging Methods in Nonlinear Dynamical Systems, Applied Mathematical Sciences 59, Springer, 1985.

[11] F. Verhulst, Nonlinear Differential Equations and Dynamical Systems, Universitext, Springer, 1991.

${ }^{1}$ UNESP-IBILCE - SÃo José do Rio Preto, SP, CEP 15054-000, BraZIL

2 Departament de Matemàtiques, Universitat Autònoma de Barcelona, 08193 Bellaterra, Barcelona, Catalonia, Spain

3 Instituto de Matemática e Estatística, Universidade Federal de Goiás, 74001-970 GoiÂnia, Goiás, BraziL

E-mail address: buzzi@ibilce.unesp.br

E-mail address: jllibre@mat.uab.cat

E-mail address: medrado@mat.ufg.br 